\title{
Brain Activity Changes in Somatosensory and Emotion-Related Areas With Medial Patellofemoral Ligament Deficiency
}

\author{
Masaru Kadowaki MD, Taku Tadenuma MD, Nobuyuki Kumahashi MD, PhD, \\ Yuji Uchio MD, PhD
}

Received: 11 February 2017/Accepted: 2 August 2017/Published online: 11 August 2017

(C) The Association of Bone and Joint Surgeons (B) 2017

\begin{abstract}
Background Patellar instability with medial patellofemoral ligament (MPFL) deficiency is a common sports injury among young people. Although nonoperative and surgical treatment can provide stability of the patella, patients often have anxiety related to the knee. We speculate that neural dysfunction may be related to anxiety in these patients; however, the mechanism in the brain that generates this anxiety remains unknown.

Questions/purposes (1) How does brain activity in patients with MPFL deficiency change in the areas related to somatic sensation against lateral shift of the patella? (2) How does patella instability, which can lead to continuous fear or apprehension for dislocation, influence brain activity in the areas related to emotion?

Methods Nineteen patients with MPFL deficiency underwent surgical reconstruction in our hospital from

Each author certifies that neither he, nor any member of his immediate family, have funding or commercial associations (consultancies, stock ownership, equity interest, patent/licensing arrangements, etc) that might pose a conflict of interest in connection with the submitted article.

All ICMJE Conflict of Interest Forms for authors and Clinical Orthopaedics and Related Research ${ }^{\mathbb{B}}$ editors and board members are on file with the publication and can be viewed on request.

Clinical Orthopaedics and Related Research ${ }^{\circledR}$ neither advocates nor endorses the use of any treatment, drug, or device. Readers are encouraged to always seek additional information, including FDAapproval status, of any drug or device prior to clinical use.

Each author certifies that his institution approved the human protocol for this investigation and that all investigations were conducted in conformity with ethical principles of research.
\end{abstract}

M. Kadowaki ( $₫)$, T. Tadenuma, N. Kumahashi, Y. Uchio Department of Orthopaedic Surgery, Shimane University, 89-1, en'ya-cho, Izumo, Shimane, Japan

e-mail: masaru11@med.shimane-u.ac.jp
April 2012 to March 2014. Excluding seven patients with osteochondral lesions, 12 patients (five males and seven females; mean age, 20 years) with MPFL deficiency were sequentially included in this study. Eleven control subjects (four males and seven females; mean age, 23 years) were recruited from medical students who had no history of knee injury. Diagnosis of the MPFL deficiency was made with MR images, which confirmed the rupture, and by proving the instability with a custom-made biomechanical device. Brain activity during passive lateral stress to the patella was assessed by functional MRI. Functional and anatomic images were analyzed using statistical parametric mapping. Differences in functional MRI outcome measures from the detected activated brain regions between the patients with MPFL deficiency and controls were assessed using t tests. Results Intergroup analysis showed less activity in several sensorimotor cortical areas, including the contralateral primary somatosensory areas (\% signal change for MPFL group $0.49 \%$ versus $1.1 \%$ for the control group; $\mathrm{p}<0.001$ ), thalamus $(0.2 \%$ versus $0.41 \%$ for the MPFL versus control, respectively; $\mathrm{p}<0.001)$, ipsilateral thalamus $(0.02 \%$ versus $0.27 \%$ for the MPFL versus control, respectively; $\mathrm{p}<$ $0.001)$, and ipsilateral cerebellum $(0.82 \%$ versus $1.25 \%$ for the MPFL versus control, respectively; $p<0.001$ ) in the MPFL deficiency group than in the control group. In contrast, the MPFL deficiency group showed more activity in several areas, including the contralateral primary motor area $(1.06 \%$ versus $0.6 \%$ for the MPFL versus control, respectively; $\mathrm{p}<0.001)$, supplementary motor area $(0.89 \%$ versus $0.52 \%$ for the MPFL versus control, respectively; $p$ $<0.001)$, prefrontal cortex $(1.09 \%$ versus $1.09 \%$ for the MPFL versus control, respectively; $\mathrm{p}<0.001)$, inferior parietal lobule $(0.89 \%$ versus $0.62 \%$ for the MPFL versus control, respectively; $\mathrm{p}<0.001)$, anterior cingulate cortex $(0.84 \%$ versus $0.08 \%$ for the MPFL versus control, 
respectively; $\mathrm{p}<0.001)$, visual cortex $(0.86 \%$ versus $0.14 \%$ for the MPFL versus control, respectively; $p<$ $0.001)$, vermis $(1.18 \%$ versus $0.37 \%$ for the MPFL versus control, respectively; $\mathrm{p}<0.001$ ), and ipsilateral prefrontal cortex $(1.1 \%$ versus $0.75 \%$ for the MPFL versus control, respectively; $\mathrm{p}<0.001)$ than did the control group.

Conclusions Less activity in the contralateral somatosensory cortical areas suggested that MPFL deficiency may lead to diminished somatic sensation against lateral shift of the patella. In contrast, increased activity in the anterior cingulate cortex, prefrontal cortex, and inferior parietal lobule may indicate anxiety or fear resulting from patellar instability, which is recognized as an aversion similar to that toward chronic pain.

Clinical Relevance This study suggests that specific brain-area activity is increased in patients with MPFL deficiency relative to that in controls. Further longitudinal research to assess brain activity and proprioception between patients pre- and postreconstructive knee surgery may reveal more regarding how patella instability is related to brain function. We hope that based on such research, a neural approach to improve patella-instability-related brain function can be developed.

\section{Introduction}

Patella dislocation and associated medial patellofemoral ligament (MPFL) deficiency is one of the most-common knee injuries in adolescents [28, 34]. Although the aim of treatment is primarily to recover knee stability, proprioception and balance have emerged as important factors to improve clinical function $[3,12,35]$. Proprioception controls apprehension regarding the joint, and is defined as the culmination of all neural inputs originating from joints, tendons, muscles, and associated deep tissue proprioceptors, which project to the central nervous system for processing and ultimately result in the regulation of reflexes and motor control. Proprioceptive stimuli are received by sensory receptors-the visual system, the vestibular system, and the peripheral mechanoreceptorswhich convert mechanical stimuli to a new action potential [16]. Mechanoreceptors, including the Pacinian corpuscle, Ruffini's end organ, and Golgi tendon end organ, have been histologically proved to exist in normal ACL fibers [32, 33]; moreover, they can be regenerated even in a reconstructed ACL [7]. These mechanoreceptors have an important role in recognizing mechanical stress to the ligament and transmitting it to the central nervous system [19].

However, the role of the MPFL in proprioception of the knee is unknown. We theorize that an MPFL deficiency could influence brain activity in somatosensory and emotional regions. First, when the patella shifts laterally with knee extension and flexion and/or quadriceps muscle contraction, the MPFL is elongated and these stimuli may be recognized and transmitted to the central nervous system by some neural organs in the MPFL and/or joint capsule and/or quadriceps muscle. Patella instability may influence this neural function followed by change in brain activity. Next, we often encounter patients who report apprehension for redislocation of the patella despite that the instability was resolved by successful conservative treatment or reconstruction surgery of the MPFL. This may be the result of the change in brain activity in the emotionrelated area. We chose functional MRI to investigate brain activity. Compared with other techniques, such as positron emission tomography or single-photon emission tomography to evaluate brain activity by detecting a transition of blood supply or metabolism of the brain, functional MRI has some advantages, including no radiation exposure, high spatial resolution, and high-contrast resolution, and can acquire images in any plane. Consequently, functional MRI has been adopted in many research areas to understand the localization of brain activity [37]. Kapreli et al. [18] reported on their evaluation of brain activity in patients with ACL injury using functional MRI.

The aim of the current study was to assess brain activity in patients with a chronic MPFL deficiency compared with control subjects who do not have a MPFL deficiency or any other knee disorders. We asked: (1) How does brain activity in patients with MPFL deficiency change in the areas related to somatic sensation against lateral shift of the patella? (2) How does patella instability, which can lead to continuous fear or apprehension for dislocation, influence brain activity in the areas related to emotion?

\section{Patients and Methods}

This controlled, prospective study was conducted in a single university from 2012 to 2014 after obtaining institutional approval by the ethics committee of the institute (approval No. 660), and written informed consent was obtained from all participants.

Twenty-nine patients with traumatic patella dislocation were seen in our hospital from April 2012 to March 2014. All MPFL ruptures were confirmed by MRI and clinically diagnosed by physical examination performed by one orthopaedic surgeon (YU). Seven patients with osteochondral lesions on the joint surface of the patella or trochlea were treated primarily by reconstructive surgery. The remaining 22 patients were first treated conservatively by fitting a brace and providing functional training of the quadriceps muscle. After less than 3 months of conservative treatment, patients who experienced redislocation or instability influencing 
their daily activities were indicated for surgical treatment. Finally, 12 patients (five males and seven females; age, $20 \pm$ 9 years; range, 15-39 years; all right handed) with chronic MPFL deficiency were sequentially included this study. Although we established the exclusion criteria of pain when the patella shifted, limited ROM in the investigated knee, or a history of previous trauma in the same leg, no patient required exclusion. Eleven medical university students without any knee disorders, including MPFL deficiency, were recruited as control subjects (four males and seven females; age, $23 \pm 4$ years; range, 19-31 years). Only righthanded subjects were recruited for controls to match the dominant hemisphere of the patient groups. We used a custom biomechanical device ("patella tester") to evaluate patella instability quantitatively. This novel device, which we designed in 2010, measures the force required to move a patella over a $5-\mathrm{mm}$ distance and calculates rigidity $(\mathrm{N} / \mathrm{mm})$ as an indicator of patella instability [10]. The test was performed by a single examiner (TM) familiar with the device.

The mean time since initial injury in the patients with MPFL deficiency was 43 months (range, 3-224 months), and the mean number of dislocations was three (range, 111). All participants felt anxiety with passive patella shift (that is, the patella apprehension test was positive). We used the Kujala score for functional assessment of a patient with a patellofemoral disorder [20]. This scoring consists of 13 questions regarding pain, physical abilities such as running and squatting, and ROM. The score ranges from 0 to 100 points, with a higher score indicating better function. At the time of enrollment, the mean Kujala score in the patient group was $69 \pm 11$ points. Patella rigidity was lower in the injured knee than in the unaffected contralateral knee in all participants (mean, $0.63 \pm 0.21 \mathrm{~N} /$ $\mathrm{mm} ; 95 \% \mathrm{CI}, 0.49-0.77)$, than on the contralateral side (mean, $1.03 \pm 0.31 \mathrm{~N} / \mathrm{mm}$; 95\% CI, 0.83-1.24) $(\mathrm{p}=0.001)$. For reference, the value of $0.95 \mathrm{~N} / \mathrm{mm}$ was obtained from healthy subjects in a previous study [21] (Table 1).

\section{Functional MRI Data Acquisition}

The functional MRI measurements were performed using a 3.0-T scanner (Signa ${ }^{\mathrm{TM}}$ HDxt; General Electric Company, Fairfield, CT, USA) with an eight-channel round head coil. First a T1-weighted sequence (repetition time [TR]/echo time $[\mathrm{TE}]=2168.96 / 9.252 \mathrm{~ms}$; matrix, $515 \times 512$; slice thickness, $6.0 \mathrm{~mm}$ ) was scanned for anatomic mapping. Then, a functional MRI series including six functional time series commenced. Sixty whole-brain echoplanar images $(\mathrm{TR} / \mathrm{TE}=3000 / 35 \mathrm{~ms}$; matrix, $64 \times 64$; slice thickness, 4.0 $\mathrm{mm})$ were acquired every 3.0 seconds.

Patients and control subjects were positioned in the supine position inside the MRI scanner. A wrap was used
Table 1. Characteristics of patients in the MPFL group

\begin{tabular}{lccccccc}
\hline $\begin{array}{l}\text { Patient } \\
\text { number }\end{array}$ & Sex & $\begin{array}{l}\text { Age } \\
\text { (years) }\end{array}$ & $\begin{array}{l}\text { Time } \\
\text { after first } \\
\text { injury } \\
\text { (months) }\end{array}$ & $\begin{array}{l}\text { Number of } \\
\text { dislocations }\end{array}$ & $\begin{array}{l}\text { Kujala } \\
\text { score }\end{array}$ & $\begin{array}{l}\text { Patella } \\
\text { rigidity (N/ } \\
\text { mm) injured } \\
\text { contralateral }\end{array}$ \\
\hline 1 & F & 18 & 6 & 1 & 84 & 0.56 & 0.87 \\
2 & M & 15 & 3 & 1 & 76 & 0.64 & 1.56 \\
3 & M & 16 & 3 & 1 & 42 & 0.64 & 1.11 \\
4 & F & 18 & 23 & 11 & 71 & 0.63 & 1.31 \\
5 & F & 15 & 21 & 2 & 66 & 0.71 & 0.91 \\
6 & M & 37 & 36 & 5 & 75 & 0.23 & 0.54 \\
7 & F & 39 & 224 & 2 & 53 & 0.98 & 1.21 \\
8 & F & 22 & 96 & 1 & 70 & 0.67 & 0.98 \\
9 & F & 15 & 47 & 5 & 75 & 0.23 & 0.50 \\
10 & F & 16 & 7 & 1 & 72 & 0.86 & 1.41 \\
11 & M & 16 & 28 & 2 & 69 & 0.62 & 1.00 \\
12 & M & 15 & 23 & 2 & 78 & 0.76 & 1.03 \\
Mean & & 20 & 43 & 3 & 69 & 0.63 & 1.04 \\
SD & & 9 & 63 & 3 & 11 & 0.21 & 0.31 \\
\hline
\end{tabular}

MPFL = medial patellofemoral ligament; $\mathrm{F}=$ female; $\mathrm{M}=$ male.

to fix their trunks to limit head motion during the stress task. Lateral stress was applied manually to the patella from the medial aspect by one examiner (MK), with the goal of gently inducing $5 \mathrm{~mm}$ of lateral displacement in as consistent a manner as possible. Both legs also were fixed with straps and pillows during the sessions. Each time series of this block design consisted of six blocks of two conditions: rest and lateral shift to the patella. Each condition lasted 30 seconds (equal to 10 whole brain images) and lateral stress to the patella was applied five times in each task phase.

Functional and anatomic images were analyzed using statistical parametric mapping (SPM8; Wellcome Trust Centre for Neuroimaging, London, UK). For each participant, slice timing of the echoplanar images time series was adjusted referring to the middle slice (the 20th slice), then realigned and resliced using the SPM8 motion correction algorithm [14] to correct the head motion accompanied by the stress task. These images were coregistered on the anatomic MRI (T1-weighted image) and were normalized to a standard brain image. The normalized echoplanar images then were processed with spatial smoothing with Gaussian filtering (8-mm full width at half maximum) [13].

\section{Statistical Analyses}

Individual statistical data analysis (first-level analysis) consisted of several steps. Detected global changes of the blood oxygenation level-dependent signal [27] were 
processed to a general linear model, which was calculated with convolution of hemodynamic response function and task block [40]. After the estimation process involving calculating the partial regression coefficient of the general linear model in all voxels, statistical analysis using a t test was performed to generate a statistical parametric map. We then could see the brain area more activated in the task phase than in the rest phase in each patient and control subject. Group analysis then (second-level analysis) was performed to find voxels in which blood oxygenation leveldependent signals were increased more substantially in the task phase than in the rest phase in each group. The statistical parametric maps of all patients and control subjects, which were generated in the individual analysis, were processed with random effects, and a one sample $t$ test was used to analyze statistical values in all voxels. The brain area activated in the task phase than in the rest phase was shown as an average for each group. In all comparisons, a probability less than 0.001 was considered as indicating statistical significance to improve the reliability of the analysis for multiple comparisons. Finally, the two groups were compared directly using a two sample t test. Brain areas of differential activation were detected between group analysis of activation as higher in patients with MPFL deficiency than in controls and as higher in controls than in patients with MPFL deficiency. The significance level of differences was a probability less than 0.001 for activation between the groups. Group analysis by SPM8 compares only statistic values-it cannot compare absolute values (the actual value of MRI signal). Therefore, the percentage of change in the MR signal in the brain area with activation was estimated using the MarsBaR SPM toolbox (http://marsbar.sourceforge.net). This process was applied to regions of interest in which we had already proved that brain activity was statistically different between the patient and control groups by SMP8, and the estimated value was not analyzed statistically.

\section{Results}

Brain activity in somatic sensation areas was lower in the MPFL deficiency group than in the control group. The areas that showed more activation in the task phase than in the rest phase are different between both groups on images of the brain map (Fig. 1). Results of comparisons between groups showed MRI signal changes in the contralateral primary sensory area (\% signal change for MPFL group, $0.49 \%$, versus $1.1 \%$ for the control group; $\mathrm{p}<0.001$ ), thalamus $(0.2 \%$ versus $0.41 \%$ for the MPFL versus control, respectively; $\mathrm{p}<0.001)$, ipsilateral thalamus $(0.02 \%$ versus $0.27 \%$ for the MPFL versus control, respectively; $\mathrm{p}<$ 0.001 , and cerebellum $(0.82 \%$ versus $1.25 \%$ for the MPFL

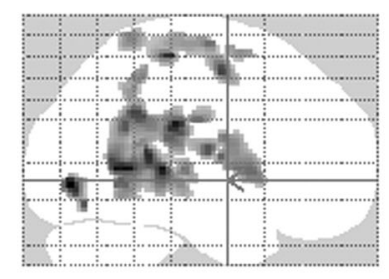

A
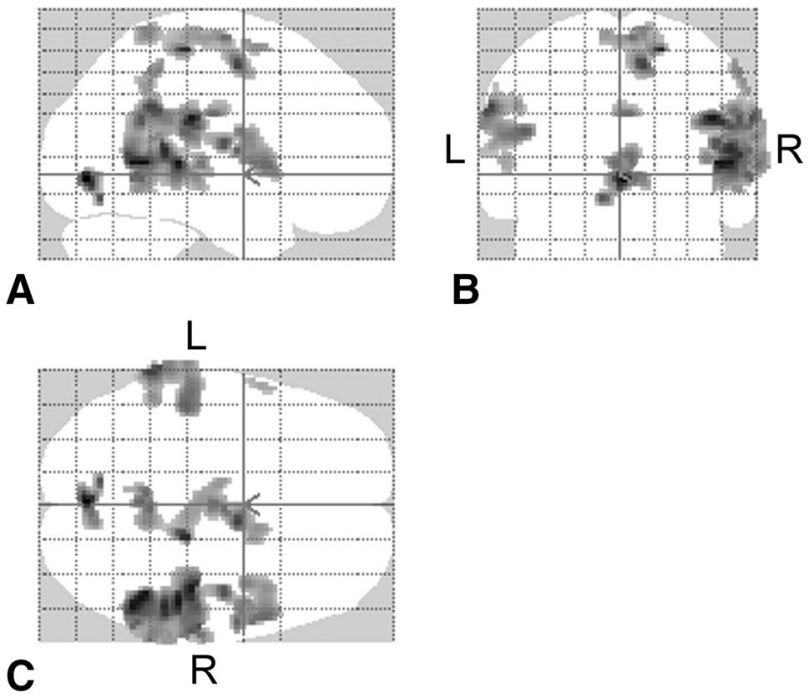

B

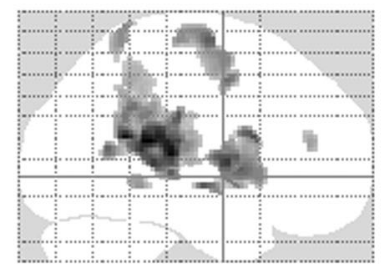

D
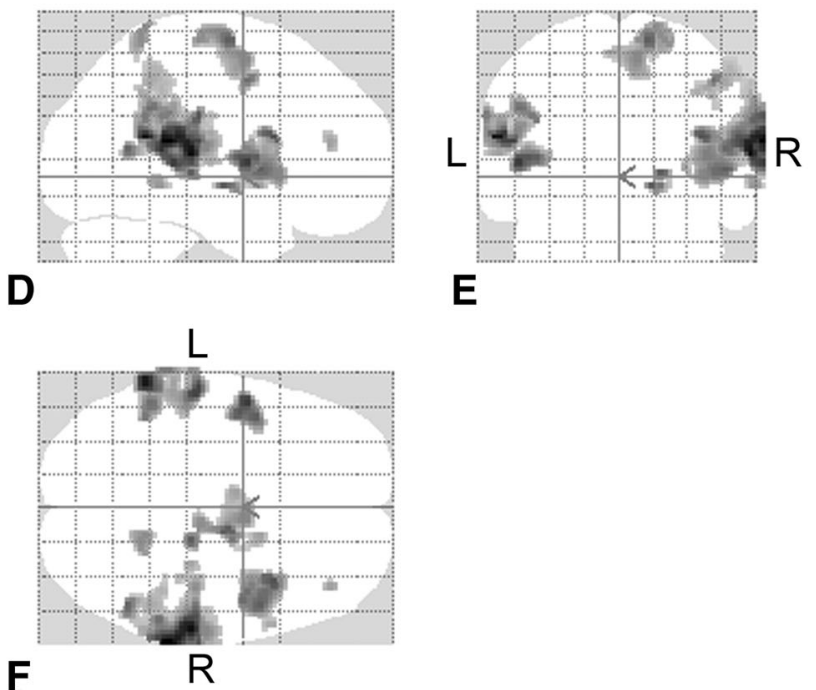

Fig 1A-F The brain area is more activated in the task phase than the rest phase as seen on the (A) sagittal, (B) coronal, and (C) horizontal views of the medial patellofemoral ligament (MPFL) deficient group than on the (D) sagittal, (E) coronal, and (F) horizontal views of the control group. In this brain map, activated areas are shown in different shades of grey. The darker grey tones have higher $\mathrm{T}$ values (which equals a lower $\mathrm{p}$ value).

versus control, respectively; $\mathrm{p}<0.001$ ) were lower in the MPFL deficiency group than in the control group (Table 2). The mean values and SD of percent signal change in these areas are shown (Fig. 2). To the contrary, brain activity was higher in the contralateral primary motor area $(1.06 \%$ versus $0.6 \%$ for the MPFL versus control, respectively; $\mathrm{p}<0.001)$, supplementary motor area $(0.89 \%$ versus $0.52 \%$ for the MPFL versus control, respectively; $p$ $<0.001)$ and visual cortex $(0.86 \%$ versus $0.14 \%$ for the MPFL versus control, respectively; $p<0.001)$ in the MPFL deficiency group than in the control group (Fig. 3).

Additionally, the areas related to emotion were activated in the MPFL deficiency group. Intergroup analysis showed 
Table 2. Brain regions showing higher functional MRI activation

\begin{tabular}{|c|c|c|c|c|c|c|c|c|c|}
\hline \multicolumn{5}{|c|}{ MPFL group $<$ control group } & \multicolumn{5}{|c|}{ MPFL group $>$ control group } \\
\hline \multirow[t]{2}{*}{ Area } & \multirow[t]{2}{*}{ MNI coordinates $(\mathrm{x}, \mathrm{y}, \mathrm{z})$} & \multirow[t]{2}{*}{$\mathrm{p}$ Value } & \multicolumn{2}{|c|}{$\begin{array}{l}\text { MRI signal change } \\
(\%)\end{array}$} & \multirow[t]{2}{*}{ Area } & \multirow[t]{2}{*}{ MNI coordinates $(\mathrm{x}, \mathrm{y}, \mathrm{z})$} & \multirow[t]{2}{*}{$\mathrm{p}$ Value } & \multicolumn{2}{|c|}{$\begin{array}{l}\text { MRI signal change } \\
(\%)\end{array}$} \\
\hline & & & MPFL & Control & & & & MPFL & Control \\
\hline \multicolumn{5}{|l|}{ Contralateral } & \multicolumn{5}{|l|}{ Contralateral } \\
\hline SM1 & $10,-50,68$ & $<0.001$ & 0.49 & 1.1 & M1 & $10,-6,52$ & $<0.001$ & 1.06 & 0.6 \\
\hline Thalamus & $2,-20,5$ & $<0.001$ & 0.2 & 0.41 & SMA & $12,4,62$ & $<0.001$ & 0.89 & 0.49 \\
\hline Ipsilateral & & & & & PF (BA44) & $40,6,6$ & $<0.001$ & 1.09 & 0.52 \\
\hline Thalamus & $-6,-24,5$ & $<0.001$ & 0.02 & 0.27 & IP (BA40) & $50,-30,26$ & $<0.001$ & 0.89 & 0.62 \\
\hline \multirow[t]{4}{*}{ Cerebellum } & $-26,-54,-22$ & $<0.001$ & 0.82 & 1.25 & $\mathrm{ACC}$ & $2,-16,31$ & $<0.001$ & 0.84 & 0.08 \\
\hline & & & & & V & $0,-78,-4$ & $<0.001$ & 0.86 & 0.14 \\
\hline & & & & & Vermis & $-6,-52,-22$ & $<0.001$ & 1.18 & 0.37 \\
\hline & & & & & PF (BA44) & $-42,5,6$ & $<0.001$ & 1.1 & 0.75 \\
\hline
\end{tabular}

MNI= Montreal Neurological Institute; MPFL = medial patellofemoral ligament; SM1 = primary sensory area; M1 = primary motor area; SMA = supplementary motor area; $\mathrm{BA}=$ Brodmann area; $\mathrm{PF}=$ prefrontal lobe; $\mathrm{IP}=$ inferior parietal lobule; $\mathrm{ACC}=$ anterior cingulate cortex; $\mathrm{V}=$ visual cortex.

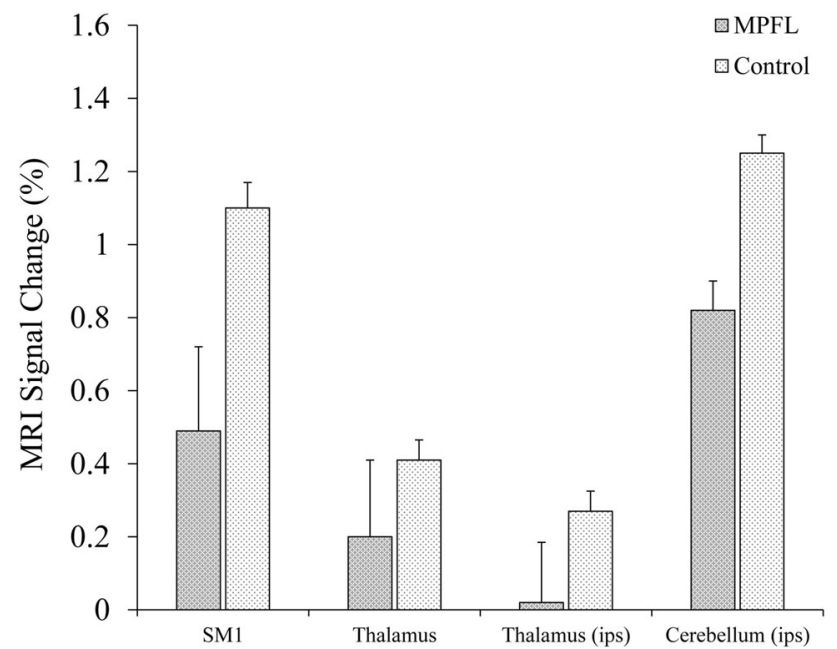

Fig. 2 The percent signal change in the area was activated higher in the medial patellofemoral ligament (MPFL) deficient group than in the control group $(\mathrm{p}<0.001)$. The mean value and SD are shown. SM1 = primary sensory area; ips = ipsilateral .

higher MRI signal changes than those in the control group in the contralateral inferior parietal lobule $(0.89 \%$ versus $0.62 \%$ for the MPFL versus control, respectively; $\mathrm{p}<$ $0.001)$, prefrontal lobe (1.09\% versus $1.09 \%$ for the MPFL versus control, respectively; $\mathrm{p}<0.001)$, anterior cingulate cortex $(0.84 \%$ versus $0.08 \%$ for the MPFL versus control, respectively; $\mathrm{p}<0.001)$, vermis $(1.18 \%$ versus $0.37 \%$ for the MPFL versus control, respectively; $\mathrm{p}<0.001$ ), and ipsilateral prefrontal lobe (Table 2). The mean value and SD of MRI signal changes are shown (Fig. 3).

\section{Discussion}

A patellar dislocation is an injury commonly sustained by young, active patients, with nearly $70 \%$ of dislocations occurring during sports activities [11, 28, 34]. The MPFL is a thin fascial band approximately $53 \mathrm{~mm}$ (range, 45-64 $\mathrm{mm})$ long [38] that links the region of the medial epicondyle of the femur to the proximal part of the medial border of the patella. Several studies have found that the MPFL is the primary passive restraint that resists lateral translation of the patella $[4,8,15,25]$. Although previous studies have shown satisfactory physical function after reconstructive surgery of the MPFL [1, 5, 6, 24], little attention has been dedicated to understanding the changes that occur in the brain. We often meet patients who report having anxiety or discomfort despite having patella stability obtained through treatment. We speculated that some changes occur in the brain activity of patients with MPFL deficiency that are related to these symptoms of anxiety or discomfort and therefore we evaluated brain activity by functional MRI in this study. Our study showed that brain activity decreased in the contralateral somatosensory cortical areas and increased in the ipsilateral anterior cingulate cortex, prefrontal cortex, and inferior parietal lobule in patients with MPFL deficiency compared with healthy control subjects. To our knowledge, this is the first study to map brain activity in patients with MPFL insufficiency.

This study has numerous limitations. First, we did not histologically prove the existence of a mechanoreceptor in MPFL. Second, actual proprioception including joint position sense or sense for angulation was not evaluated, 
Fig. 3 The percent signal change in the area was activated higher in the control than in the medial patellofemoral ligament (MPFL) deficient group ( $\mathrm{p}<$ 0.001 ). The mean value and SD are shown. $\mathrm{M} 1$ = primary motor area; SMA = supplementary motor area; $\mathrm{PF}=$ prefrontal lobe; IP = inferior parietal lobe; $\mathrm{ACC}=$ anterior cingulate cortex; V= visual cortex; ips = ipsilateral.

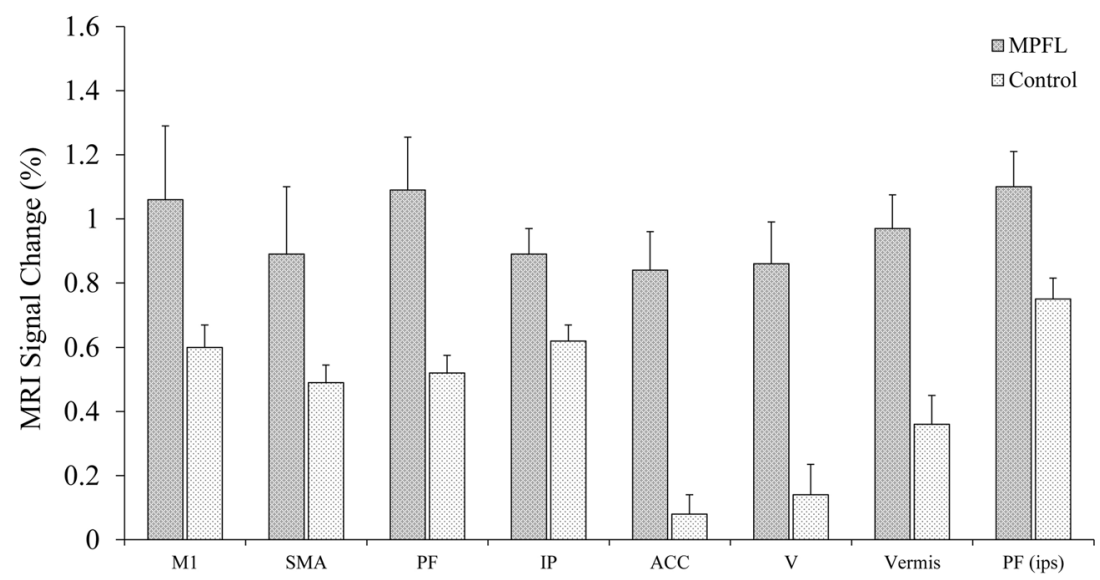

and on these points, we could not determine the contribution or causation of MPFL deficiency to brain activity. Further studies to establish these aspects are required to validate the results of this study. There were also some limitations related to the analysis method used. Although 12 patients were regarded as sufficient for analysis using the SPM software, a greater number would be required to apply the results to all other patients with MPFL deficiency. The small number of patients and control subjects in this study could have caused biases related to the demographic characteristics of the patients, such as sex, number of dislocations, and posttraumatic time. Additionally, because this was not a randomized controlled study, differences in the initial somatosensory or physical function and dominant leg could have affected the results. Finally, the applied lateral stress to the patella might not have been constant for all patients and control subjects because the stress was generated manually, so devices that can apply constant stress should be considered in future experiments to provide more reliable results. In this study, the examiner did his best to provide sufficient force to displace the patella by $5 \mathrm{~mm}$, and no further.

Our study showed the differences in brain activity of patients with MPFL deficiency compared with that of healthy control subjects. Similar to those with ACL deficiency [18], patients with MPFL deficiency showed diminished activation in the primary sensory area and thalamus, both of which play leading roles in input from proprioception in normal knees. The decreased activation of these cortical areas in the MPFL group could be attributed to the differentiation of the ascending afferent pathway from the injured knee. We propose some theories regarding the possible response to this incitement. One is that the MPFL has a function as a mechanoreceptor, which could detect a lateral shift of the patella when the MPFL was elongated or shortened. However, a histologic study by Tuxoe et al. [38] showed that the MPFL had no mechanoreceptors but did have nerve fibers, which seems to deny this theory. Another theory is that the Golgi tendon end organ or muscle spindle senses the elongation or shortening of the patella tendon and/or quadriceps femoris muscle and/or joint capsule. We surmise that the reasons for altered brain activity in patients with MPFL deficiency is that the sensory function of tendons, joint capsule, and muscles may be affected by patella instability. Diminished activity of the ipsilateral cerebellum indicates a decreased ascending afferent pathway through the spinocerebellar tract, which is one of the somatosensory pathways. This finding also could show function of proprioception in the MPFL-injured knee. We cannot extrapolate further owing to the lack of studies on the neural function of the MPFL, although the brain activity of areas related to somatosensory pathways was clearly less in patients with MPFL deficiency than in the control subjects.

Another interesting finding of our study was the increased activity of the primary motor area, supplementary motor area, prefrontal lobe (Brodmann area [BA] 44), inferior parietal lobule (BA40), and anterior cingulate cortex. A meta-analysis showed that the supplementary motor area, anterior cingulate cortex, prefrontal lobe, inferior parietal lobule, and vermis respond to pain in healthy subjects [29]. These areas may play a role with respect to pain; the anterior cingulate cortex, especially, is well known as an area composed of the so-called "pain matrix" [36]. Pain has been recognized not just as a physical sensation, but also as an integration of several emotions [22]. It has been confirmed that discomfort feelings such as dissatisfaction, a sense of alienation, sorrow, and envy can activate the pain-related brain area $[9,26,30]$. This means the pain matrix is not exclusively specific for pain, but also for detection of the salient sensory input and should thus be called the "neuromatrix." Although we did not apply pain, but rather lateral shift of the patella, participants may feel discomfort such as 
apprehension or fear related to patella instability. These emotions could activate the neuromatrix. It has been reported that such areas could increase activation in pathologic conditions. Baliki et al. [2] reported that anterior cingulate cortex activity increased with spontaneous pain in patients with chronic back pain. Ikemoto et al. [17] reported that activity in the primary motor area, supplementary motor area, anterior cingulate cortex, and cerebellum increased with noxious stimulation in patients with neuropathic pain (allodynia). May et al. [23] reported that activity in the supplementary motor area and cerebellum increased with headache attacks in patients with cluster headaches. Ushida et al. [39] reported that the anterior cingulate cortex was activated in a patient with hand allodynia by just watching a movie where a hand was touched with a brush. Thus, increased activity in the primary motor area, supplementary motor area, anterior cingulate cortex, and cerebellum, as seen in our patients, may not indicate a normal response to a lateral shift of the patella. We were concerned that apprehension regarding dislocation could be imprinted as a fear memory resulting from recurrent dislocation or a long posttraumatic interval in patients with MPFL deficiency. If the apprehension can become chronic, like pain, it may lead to persistent feelings of anxiety or instability, even with a stabilized knee after MPFL reconstruction.

Increased activation in the visual cortex against decreased activation in the primary sensory area and thalamus was seen in the MPFL group, indicating modifications of the central nervous system. A previous study of ACL injury suggested that the higher activation of this area was the result of its increased need for motion visualization and feedback during the movement of injured joints owing to restrictive proprioceptive information [18], which was supported by the fact that visual and proprioceptive information were combined to control limb movements [31]. Because diminished somatosensory function such as position sense also required additional support from the visual sense (determined by the Romberg test), this finding may show compensation for hypoactive proprioception resulting from MPFL deficiency.

We found that brain activity in patients with MPFL deficiency differed from that of control subjects in the patellar apprehension test. This may suggest functional depression of proprioception in the knee and implies the need for additional evaluation of joint position sense or sense for angulation. Because brain reorganization occurred in patients with MPFL deficiency, future studies should focus on rehabilitation for reeducation of the central nervous system-that is, neurocognitive rehabilitation-rather than focusing only on the peripheral neuromuscular function. Whether treated conservatively or surgically, it is important to avoid recurrent dislocation, which could enhance fear or anxiety in the patient. Further research is needed to clarify how to manage MPFL deficiency and to improve clinical outcomes from a neurologic viewpoint.

Acknowledgments We thank Shinji Hara (radiologic technician, Department of Radiology, Shimane University Hospital) and Takuya Matsumoto (physical therapist, Department of Rehabilitation, Shimane University Hospital) who contributed to our research.

\section{References}

1. Astur DC, Gouveia GB, Borges JH, Astur N, Arliani GG, Kaleka CC, Cohen M. Medial patellofemoral ligament reconstruction: a longitudinal study comparison of 2 techniques with 2 and 5-years follow-up. Open Orthop J. 2015;9:198-203.

2. Baliki MN, Geha PY, Jabakhanji R, Harden N, Schnitzer TJ, Apkarian AV. A preliminary fMRI study of analgesic treatment in chronic back pain and knee osteoarthritis. Mol Pain. 2008;4:47.

3. Barrett DS. Proprioception and function after anterior cruciate reconstruction. J Bone Joint Surg Br. 1991;73:833-837.

4. Conlan T, Garth WP Jr, Lemons JE. Evaluation of the medial soft-tissue restraints of the extensor mechanism of the knee. $J$ Bone Joint Surg Am. 1993;75:682-693.

5. Csintalan RP, Latt LD, Fornalski S, Raiszadeh K, Inacio MC, Fithian DC. Medial patellofemoral ligament (MPFL) reconstruction for the treatment of patellofemoral instability. J Knee Surg. 2014;27:139-146.

6. Deie M, Ochi M, Adachi N, Shibuya H, Nakamae A. Medial patellofemoral ligament reconstruction fixed with a cylindrical bone plug and a grafted semitendinosus tendon at the original femoral site for recurrent patellar dislocation. Am J Sports Med. 2011;39:140-145.

7. Denti M, Monteleone M, Berardi A, Panni AS. Anterior cruciate ligament mechanoreceptors: histologic studies on lesions and reconstruction. Clin Orthop Relat Res. 1994;308:29-32.

8. Desio SM, Burks RT, Bachus KN. Soft tissue restraints to lateral patellar translation in the human knee. Am J Sports Med. 1998;26:59-65.

9. Eisenberger NI, Lieberman MD, Williams KD. Does rejection hurt? An FMRI study of social exclusion. Science. 2003;302:290-292.

10. Egusa N, Mori R, Uchio Y. Measurement characteristics of a force-displacement curve for chronic patellar instability. Clin J Sport Med. 2010;20:458-463.

11. Fithian DC, Paxton EW, Stone ML, Silva P, Davis DK, Elias DA, White LM. Epidemiology and natural history of acute patellar dislocation. Am J Sports Med. 2004;32:1114-1121.

12. Fremerey RW, Lobenhoffer P, Zeichen J, Skutek M, Bosch U, Tscherne $\mathrm{H}$. Proprioception after rehabilitation and reconstruction in knees with deficiency of the anterior cruciate ligament: a prospective, longitudinal study. $J$ Bone Joint Surg $\mathrm{Br}$. 2000;82:801-806.

13. Friston KJ, Josephs O, Zarahn E, Holmes AP, Rouquette S, Poline J. To smooth or not to smooth? Bias and efficiency in fMRI time-series analysis. Neuroimage. 2000;12:196-208.

14. Friston KJ, Williams S, Howard R, Frackowiak RS, Turner R. Movement-related effects in fMRI time-series. Magn Reson Med. 1996;35:346-355.

15. Hautamaa PV, Fithian DC, Kaufman KR, Daniel DM, Pohlmeyer AM. Medial soft tissue restraints in lateral patellar instability and repair. Clin Orthop Relat Res. 1998;349:174-182. 
16. Hewett TE, Paterno MV, Myer GD. Strategies for enhancing proprioception and neuromuscular control of the knee. Clin Orthop Relat Res. 2002;402:76-94.

17. Ikemoto T. Ushida T, Tanaka S, Morio K, Zinchuk V, Tani T, Taniguchi S, Ushida A. Painful mechanical stimulation evokes activation of distinct functional areas in the brain: comparison of normal subjects and two patients with neuropathic pain. Pain Res. 2003;18:137-144. Available at: https://www.jstage.jst.go.jp/ article/pain/18/3/18_4/_pdf. Accessed July 14, 2017.

18. Kapreli E, Athanasopoulos S, Gliatis J, Papathanasiou M, Peeters R, Strimpakos N, Van Hecke P, Gouliamos A, Sunaert S. Anterior cruciate ligament deficiency causes brain plasticity: a functional MRI study. Am J Sports Med. 2009;37:2419-2426.

19. Kennedy JC, Alexander IJ, Hayes KC. Nerve supply of the human knee and its functional importance. Am J Sports Med. 1982;10:329-335.

20. Kujala UM, Jaakkola LH, Koskinen SK, Taimela S, Hurme M, Nelimarkka O. Scoring of patellofemoral disorders. Arthroscopy. 1993;9:159-163.

21. Kumahashi N, Kuwata S, Takuwa H, Egusa N, Uchio Y. Longitudinal change of medial and lateral patellar stiffness after reconstruction of the medial patellofemoral ligament for patients with recurrent patellar dislocation. J Bone Joint Surg Am. 2016;98:576-583.

22. Lieberman MD, Eisenberger NI. Neuroscience: pains and pleasures of social life. Science. 2009;323:890-891.

23. May A, Bahra A, Büchel C, Frackowiak RS, Goadsby PJ. Hypothalamic activation in cluster headache attacks. Lancet. 1998;352:275-278.

24. Mulliez A, Lambrecht D, Verbruggen D, Van Der Straeten C, Verdonk P, Victor J. Clinical outcome in MPFL reconstruction with and without tuberositas transposition. Knee Surg Sports Traumatol Arthrosc. 2015 Jun 2. [Epub ahead of print]

25. Nomura E, Horiuchi Y, Kihara M. Medial patellofemoral ligament restraint in lateral patellar translation and reconstruction. Knee. 2000;7:121-127.

26. O'Connor MF, Wellisch DK, Stanton AL, Eisenberger NI, Irwin MR, Lieberman MD. Craving love? Enduring grief activates brain's reward center. Neuroimage. 2008;42:969-972.
27. Ogawa S, Lee TM, Kay AR, Tank DW. Brain magnetic resonance imaging with contrast dependent on blood oxygenation. Proc Natl Acad Sci U S A. 1990;87:9868-9872.

28. Palmu S, Kallio PE, Donell ST, Helenius I, Nietosvaara Y. Acute patellar dislocation in children and adolescents: a randomized clinical trial. J Bone Joint Surg Am. 2008;90:463-470.

29. Peyron R, Laurent B, García-Larrea L. Functional imaging of brain responses to pain: a review and meta-analysis (2000). Neurophysiol Clin. 2000;30:263-288.

30. Sanfey AG, Rilling JK, Aronson JA, Nystrom LE, Cohen JD. The neural basis of economic decision-making in the ultimatum game. Science. 2003;300:1755-1758.

31. Scheidt RA, Conditt MA, Secco EL, Mussa-Ivaldi FA. Interaction of visual and proprioceptive feedback during adaptation of human reaching movements. J Neurophysiol. 2005;93:3200-3213.

32. Schultz RA, Miller DC, Kerr CS, Micheli L. Mechanoreceptors in human cruciate ligaments: a histological study. J Bone Joint Surg Am. 1984;66:1072-1076.

33. Schutte MJ, Dabezies EJ, Zimny ML, Happel LT. Neural anatomy of the human anterior cruciate ligament. J Bone Joint Surg Am. 1987;69:243-247.

34. Shea K, Nilsson K, Belzer J. Patellar dislocation in skeletally immature athletes. Oper Tech Sports Med. 2006;14:188-196.

35. Shiraishi M, Mizuta H, Kubota K, Otsuka Y, Nagamoto N, Takagi K. Stabilometric assessment in the anterior cruciate ligament-reconstructed knee. Clin J Sport Med. 1996;6:32-39.

36. Tracey I, Mantyh PW. The cerebral signature for pain perception and its modulation. Neuron. 2007;55:377-391.

37. Turner R, Jones T. Techniques for imaging neuroscience. Br Med Bull. 2003;65:3-20.

38. Tuxoe JI, Teir M, Winge S, Nielson PL. The medial patellofemoral ligament: a dissection study. Knee Surg Sports Traumatol Arthrosc. 2002;10:138-140.

39. Ushida T, Ikemoto T, Taniguchi S, Ishida K, Murata Y, Ueda W, Tanaka S, Ushida A, Tani T. Virtual pain stimulation of allodynia patients activates cortical representation of pain and emotions: a functional MRI study. Brain Topogr. 2005;18:27-35.

40. Worsley KJ, Friston KJ. Analysis of fMRI time-series revisited: again. Neuroimage. 1995;2:173-181. 